\title{
SOME NEW RESULTS OF TWO OPEN PROBLEMS RELATED TO INTEGRAL INEQUALITIES
}

\author{
ARTION KASHURI AND ROZANA LIKO
}

Abstract. In this paper, we have solved two open problems, and as consequence some interesting integral inequalities are obtained.

Mathematics subject classification (2010): 26D15.

Keywords and phrases: Integral inequality, convex functions.

\section{REFERENCES}

[1] W. J. LiU, G. S. Cheng, C. C. LI, Further development of an open problem concerning an integral inequality, JIPAM. J. Inequal. Pure Appl. Math., ISSN: 1443-5756, 9, 1 (2008), Art. 14.

[2] W. LiU, Q. NGO, V. HuY, Several interesting integral inequalities, Journal of Mathematical Inequalities, ISSN: 1846-579X (print), 1848-9575 (online), 3, 2 (2009), 201-212. 